\title{
VCP, a Major ATPase in the Cells, as a Novel Drug Target for Currently Incurable Disorders
}

\author{
Akira Kakizuka
}

Abstract Neuroprotection would be a novel therapeutic strategy for the prevention or retardation of clinical manifestations of currently incurable eye diseases as well as neurodegenerative diseases. A decrease in cellular ATP levels may contribute to the pathologies of these diseases; therefore, stabilization of ATP levels may retard the disease progression. We created novel small compounds (Kyoto University Substances, KUSs) to inhibit the ATPase activity of VCP (valosin-containing protein), the most abundant soluble ATPase in the cell. KUSs did not apparently impair the reported cellular VCP functions. Nevertheless, they significantly suppressed the VCP-dependent decrease of cellular ATP levels. Moreover, KUSs as well as exogenous ATP or ATP-producing compounds suppressed endoplasmic reticulum (ER) stress, and indeed protected various types of cultured cells from cell deathinducing insults. We then examined the efficacies of KUSs in rd10, a mouse model of retinitis pigmentosa. KUSs not only prevented photoreceptor cell death but also preserved visual function. These results reveal an unexpected, crucial role of ATP consumption by VCP for the determination of cell fate in the pathological context, and point to a promising new neuroprotective strategy for currently incurable eye and neurodegenerative diseases.

Keywords VCP $\bullet$ ATP regulation $\bullet$ Incurable diseases $\bullet$ Retinitis pigmentosa $\bullet$ ER stress $\bullet$ Cell death $\bullet$ Drug discovery

\section{Background}

Despite recent advances in medical care, there remain many incurable disorders, e.g. neurodegenerative diseases, incurable eye diseases, etc. In these disorders, the major pathology is early cell death in the affected organs, which precedes the death of the individual, often affecting the quality of life over extended periods.

\footnotetext{
A. Kakizuka, M.D., Ph.D. ( $\square)$

Laboratory of Functional Biology, Kyoto University Graduate School of Biostudies, Kyoto 606-8501, Japan

e-mail: kakizuka@lif.kyoto-u.ac.jp 
To date, no drug has been successful at inhibiting or delaying such early cell death in vivo. In the 1990s, with much optimism, caspase inhibitors were developed and were expected to be such miracle drugs, but they were not able to prevent cell death in vivo. Indeed, later research revealed that caspases determine how cells die but are not able to affect the commitment to cell death.

Retinitis pigmentosa, in which a gradual degeneration and loss of photoreceptors occur along with aging, causes severe visual deterioration, and more than 1.5 million patients worldwide are estimated to be suffering from this eye disorder. There is no effective treatment that can prevent or delay the symptoms of retinitis pigmentosa. At least 60 genes have been implicated in retinitis pigmentosa, and therefore the cellular etiology - that is, the constellation of cellular mishaps that culminate in cell death - is the subject of ongoing debate. Involvement of endoplasmic reticulum (ER) stress has been proposed as a pathological mechanism in retinitis pigmentosa $[1,2]$.

\section{VCP in Neurodegenerative Disorders}

Using polyglutamine disease models, we have long been searching for common molecular bases in neurodegenerative diseases, and have found valosin-containing protein (VCP), an AAA (ATPases Associated with diverse cellular Activities)-type ATPase with ubiquitous expression, as a major player causing neurodegeneration. VCP is a phylogenetically well-conserved protein, and the respective amino acid sequences are identical among mouse, rat, and human, and $84 \%$ identical between human and Drosophila [3]. To search for modifier genes that are involved in the pathogenesis of polyglutamine diseases, we first created Drosophila models of polyglutamine diseases, and then performed genetic analyses. In 2002, our mutant screening revealed that Ter94 loss-of-function alleles mitigated eye degeneration that was induced by the expression of genes with expanded polyglutamine tracts [3]. Consistent with these results, overexpression of wild-type Ter94 exacerbated the polyglutamine-induced eye degeneration [3]. It is notable that the mammalian Ter94 ortholog is $V C P$. Thus, these results implicated VCP in the pathogenesis of human neurodegenerative diseases [4]. In 2004 and 2010, VCP mutations were identified that are causative for IBMPFD (inclusion body myopathy associated with Paget disease of bone and frontotemporal dementia) [5], a human hereditary disease with dementia, and for rare cases of familial amyotrophic lateral sclerosis (ALS) [6], respectively. In our evaluation, all tested pathogenic VCPs showed significant elevations of the ATPase activities, as compared with wild-type VCP [7], and thus we proposed the possibility that the constitutive elevation of its ATPase activity is a pathogenic mechanism.

These lines of evidence suggested that specific inhibitors of the ATPase activity of VCP could protect neuronal cells. In addition to its ATPase activity, however, $\mathrm{VCP}$ has been proposed to act in many important cellular processes [8-10], e.g., proteasome-mediated protein degradation, endoplasmic reticulum-associated degradation (ERAD), cell cycle control, membrane fusion, maintenance of the 
Golgi apparatus, protein trafficking, autophagy, genomic DNA surveillance, etc. Indeed, VCP knockdown and overexpression of dominant-negative forms of VCP induced cell death in essentially all cultured cells $[11,12]$. DBeQ, a recently reported VCP inhibitor (with an in vitro IC50 of $1 \mu \mathrm{M}$ ) [13], also induced cell death. Among the diverse cellular functions of VCP, some would require ATP hydrolysis and others would not. Thus, we believed it would be possible to find or develop small compounds that can inhibit or reduce the ATPase activity of VCP without inducing cellular toxicity.

\section{Development of Novel Inhibitors (KUSs) of VCP ATPase Activity That Do Not Block VCP Cellular Functions}

In our screening for novel VCP ATPase inhibitors, we found that a naphthalene derivative can inhibit the ATPase activity of VCP without showing apparent toxicity in cultured cells. Via the modification of its chemical structure, we created about 200 new compounds and named them Kyoto University Substances (KUSs). Some of them clearly inhibited the ATPase activity of recombinant VCP in vitro with $\mathrm{IC}_{50}$ values ranging from approximately $100 \mathrm{nM}$ to $1 \mu \mathrm{M}$ (Fig. 1). Importantly, the KUSs did not appear to inhibit the ATPase activity of N-ethylmaleimide-sensitive fusion protein (NSF), the protein most closely related to VCP.

As reported, DBeQ induced accumulation of ubiquitinated proteins, ER stress, autophagy, and eventually cell death. In contrast, KUSs (e.g. KUS31, 69, 94, 121, and 187) did not induce any of these phenotypes. These results clearly indicated that ATPase inhibition by KUS31, 69, 94, 121, and 187 (referred to as "KUSs" hereafter) did not interfere with reported cellular VCP functions (referred to as "VCP functions" hereafter). These results indicate that VCP functions do not necessarily require its ATPase activity.
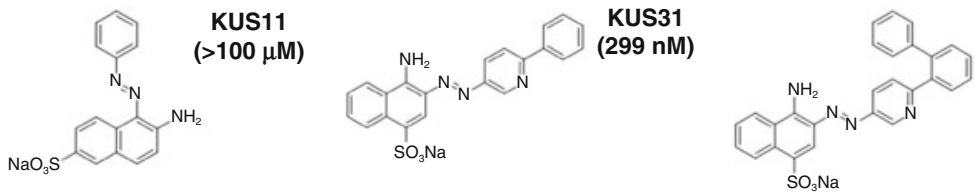

KUS69

$(116 \mathrm{nM})$
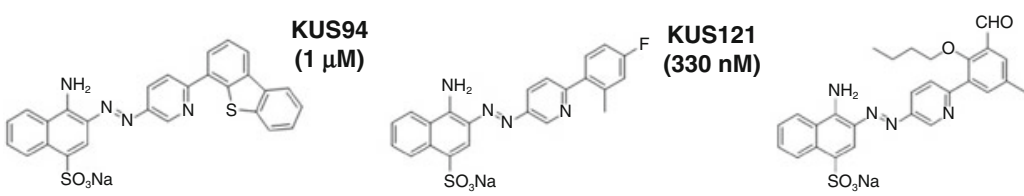

KUS187

(186 nM)

Fig. 1 Structures and $\mathrm{IC}_{50}$ values of Kyoto University Substances (KUSs), novel valosincontaining protein (VCP) modulators (Modified from Ikeda et al. [22]). Structures and $\mathrm{IC}_{50}$ values of KUS11, KUS31, KUS69, KUS94, KUS121, and KUS187 are shown. Note that KUS11 did not inhibit the ATPase activity of recombinant VCP, and it did not share a common structure with the other KUSs 


\section{KUSs Protected Cells Under ER Stress-Inducing Conditions}

Over and above their lack of toxicity, KUSs were found to protect cells from several cell death-inducing insults. For example, HeLa cells died within several days when cultured under low-glucose conditions ( $0.2 \mathrm{~g} / \mathrm{l}$ of glucose) (Fig. 2a). However, the addition of KUSs prevented cell death under these conditions (Fig. 2a, b). Similar protective effects were also observed when HeLa cells were cultured with tunicamycin (Tm) (Fig. 2c), or when HEK293 cells were cultured under serum-free conditions (Fig. 2d). These data indicated that inhibition of VCP ATPase activity by KUSs was beneficial for cells against cell death-inducing insults.

Tunicamycin treatment and glucose starvation cause ER stress and eventually lead to cell death. C/EBP-homologous protein (CHOP) is upregulated during ER stress, and is proposed to mediate the ER stress-induced cell death [14]. Indeed, the induction of CHOP in tunicamycin-treated HeLa cells was suppressed by the addition of KUSs. The induction of $78 \mathrm{kDa}$ glucose-regulated protein (Grp78), another ER stress marker [15], was also suppressed by KUSs in the tunicamycin-treated cells.
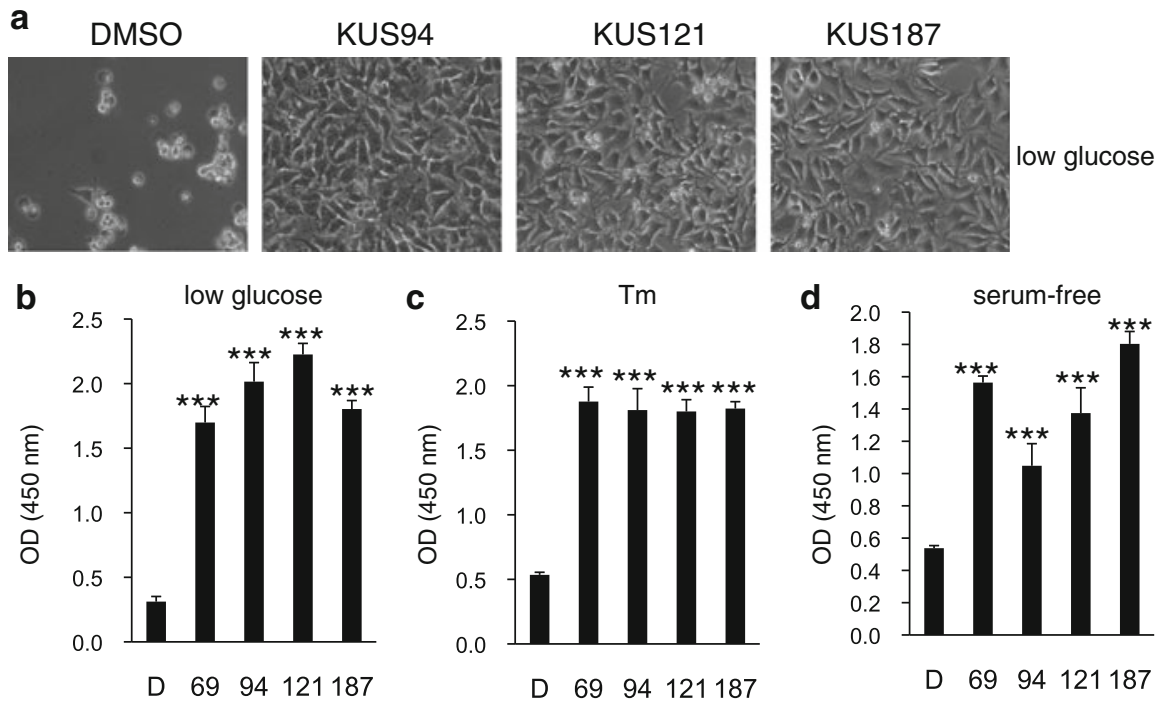

Fig. 2 Prevention of cell death and endoplasmic reticulum (ER) stress by Kyoto University Substances (KUSs) (Modified from Ikeda et al. [22]). (a) Photographs of HeLa cells, cultured with DMSO (DMSO) or KUSs (KUS94, KUS121, and KUS187, $20 \mu \mathrm{M}$ each) for $41 \mathrm{~h}$ in low-glucose $(0.2 \mathrm{~g} / \mathrm{l})$ medium. Scale bar, $100 \mu \mathrm{m}$. (b, c) WST (water-soluble tetrazolium salt) values reflecting relative live cell numbers are shown as optical density (OD) at $450 \mathrm{~nm}$. Error bars indicate standard deviations. (b) WST values of HeLa cells, cultured in low glucose (0.2 g/l) with DMSO (D) or KUSs (50 $\mu \mathrm{M}$ for KUS121; $20 \mu \mathrm{M}$ for KUS69, KUS94, and KUS187; $n=3$ ) for $41 \mathrm{~h}$. (c) Cell viability, indicated by WST values of HeLa cells, cultured with tunicamycin $(\mathrm{Tm})(0.2 \mu \mathrm{g} / \mathrm{ml})$ for $41 \mathrm{~h}$ with DMSO (D) or KUSs $(20 \mu \mathrm{M}$ each; $n=3)$. (d) WST values of HEK293 cells, cultured under serum-free conditions for $65 \mathrm{~h}$ with DMSO (D) or KUSs $(20 \mu \mathrm{M}$ each; $n=3)$. *** $P<0.001$ vs. DMSO control (Dunnett's test) 
These data indicate that KUSs have the ability to suppress ER stress and to promote cell survival in certain conditions.

We next examined the effect of KUSs on cellular ATP levels. After a $20 \mathrm{~h}$ incubation in low-glucose medium $(0.25 \mathrm{~g} / \mathrm{l})$, glucose levels in the medium approached zero, and ATP levels in the cells (control cells) also significantly decreased. In contrast, ATP levels in cells cultured in low-glucose medium and KUSs remained significantly higher than those in the control cells.

\section{KUSs or Exogenous ATP Prevented ER Stress in Cultured Cells}

ER stress has been believed to be induced by the accumulation of misfolded proteins, or protein aggregates, in the ER [16-18]. We recently identified laminin $\gamma 1$ as an aggregation-prone protein in the ER. We therefore examined the change of laminin $\gamma 1$ states in ER stress by tunicamycin treatments. Expression of laminin $\gamma 1$ was diffusely observed throughout the ER in normal cells (Control in Fig. 3a). In tunicamycin-treated cells, laminin $\gamma 1$ formed clear aggregates (DMSO in Fig. 3a). However, $50 \mu \mathrm{M}$ KUSs (KUS69, 94, 121, and 187) or $1 \mathrm{mM}$ ATP treatments prevented its aggregation (Fig. 3a). The KUSs or ATP treatment similarly prevented decreases of ATP levels in tunicamycin-treated cells (Fig. 3b). By contrast, KUS11, which could not inhibit VCP ATPase, was unable to prevent the tunicamycinelicited drop in cellular ATP concentration (Figs. 1 and 3b). The addition of $0.1 \mathrm{mM}$ ATP or 3 to $10 \mathrm{mM}$ methylpyruvate (weakly membrane-permeable pyruvate, which is converted to ATP in mitochondria) was also ineffective in preventing the aggregation of laminin $\gamma 1$ in tunicamycin-treated cells (Fig. 3a), but could nevertheless suppress the induction of ER stress, namely CHOP induction (Fig. 3c). These results indicated that the ER is more sensitive to decreases in ATP levels than to the presence of aggregates.

\section{KUSs Mitigated Pathologies of rd10, a Mouse Model of Retinitis Pigmentosa}

We have long been seeking a new strategy to protect retinal neuronal cells. In retinitis pigmentosa, an involvement of ER stress has been proposed [1, 2]. We wondered whether the protective effects of KUSs in cell culture would translate in vivo to the prevention of degeneration of photoreceptor cells in $\mathrm{rd} 10$ mice, a representative mouse model of retinitis pigmentosa [19]. Rd10 mice carry a mutation in a gene encoding the rod cyclic guanosine monophosphate (cGMP) phosphodiesterase beta subunit (PDE6B) [19]. Similar mutations are found in patients with retinitis pigmentosa. 
a
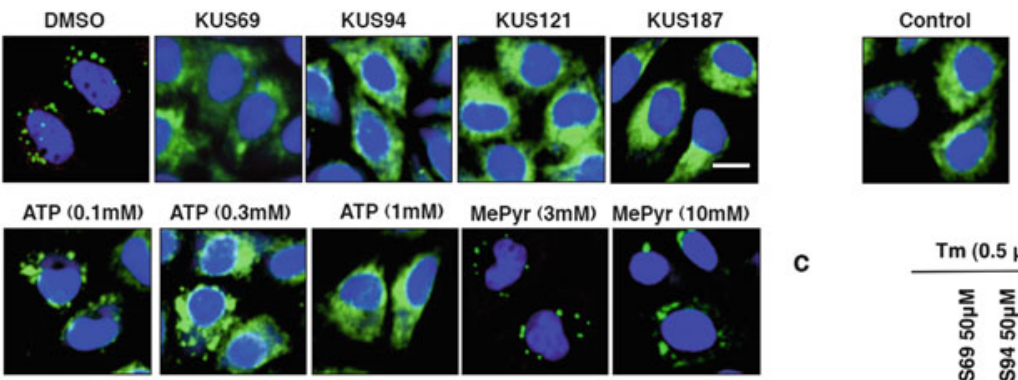

ATP (1mM)

MePyr (3mM) MePyr (10mM)
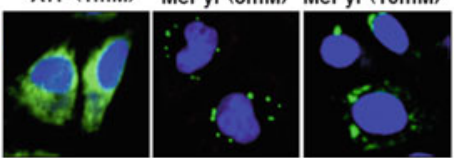

c

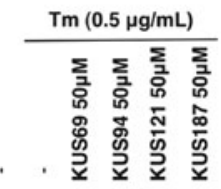

b
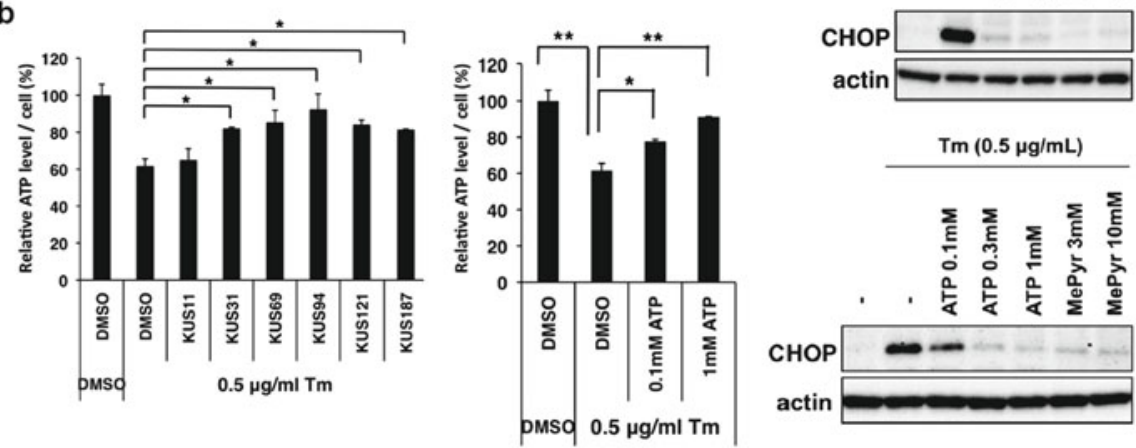

$\operatorname{Tm}(0.5 \mu \mathrm{g} / \mathrm{mL})$

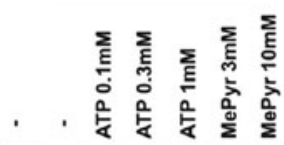

CHOP

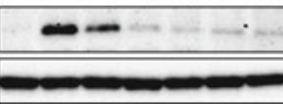

Fig. 3 Kyoto University Substances (KUSs) and ATP each prevented a decrease of ATP levels and ameliorated endoplasmic reticulum (ER) stress in tunicamycin (Tm)-treated cells (Modified from Ikeda et al. [22]). (a) Immunocytochemical analyses of HeLa cells by an anti-laminin $\gamma 1$ antibody. HeLa cells were treated with $0.5 \mu \mathrm{g} / \mathrm{ml}$ of Tm for $5 \mathrm{~h}$ in the presence of KUSs $(50 \mu \mathrm{M})$, ATP $(0.1$, 0.3 , and $1 \mathrm{mM}$ ), methylpyruvate (MePyr) $(3$ and $10 \mathrm{mM})$, or vehicle alone (DMSO). Then, cells were fixed and subjected to immunocytochemical analyses. Normally growing HeLa cells were also analyzed (Control). Scale bar, $10 \mu \mathrm{m}$. (b) Measurements of the relative amounts of ATP per cell. HeLa cells were treated with Tm $(0.5 \mu \mathrm{g} / \mathrm{ml})$ for $24 \mathrm{~h}$ in the presence of KUSs $(50 \mu \mathrm{M})$ or ATP $(0.1$ and $1 \mathrm{mM})$, or vehicle alone (DMSO), and were harvested. Then, ATP amounts from $1.5 \times 10^{5}$ cells were measured. $* P<0.05, * * P<0.01$. Error bars indicate standard deviations. (c) Western blot analyses of C/EBP-homologous protein (CHOP). HeLa cells were treated with $0.5 \mu \mathrm{g} / \mathrm{ml}$ of Tm for $5 \mathrm{~h}$ in the presence of KUSs $(50 \mu \mathrm{M})$, ATP $(0.1,0.3$, and $1 \mathrm{mM}), \operatorname{MePyr}(3$ and $10 \mathrm{mM})$, or vehicle alone (-). Then, cells were harvested and subjected to western blot analyses. Actin served as a loading control

Starting 7 days after birth, KUS121 or KUS187 was administered daily (50 mg/kg) by intraperitoneal injection. Spectral-domain optical coherence tomography (SD-OCT) examination showed that at age 25 days, the thinning of the outer nuclear layer (ONL) was clearly observed in the control rd10 mice (Fig. 4a). The ONL and the junction line between the inner segment and outer segment (arrow heads in Fig. 4a), which is generally considered to be positively associated with visual function $[20,21]$, were clearly detected in the KUS-treated rd10 mice but not in control rd10 mice. A very small electroretinogram response was observed in control rd10 mice, but an almost normal electroretinogram response was observed in most of the KUS-treated rd10 mice (Fig. 4b). At age 29 days, the photoreceptor layer was barely 


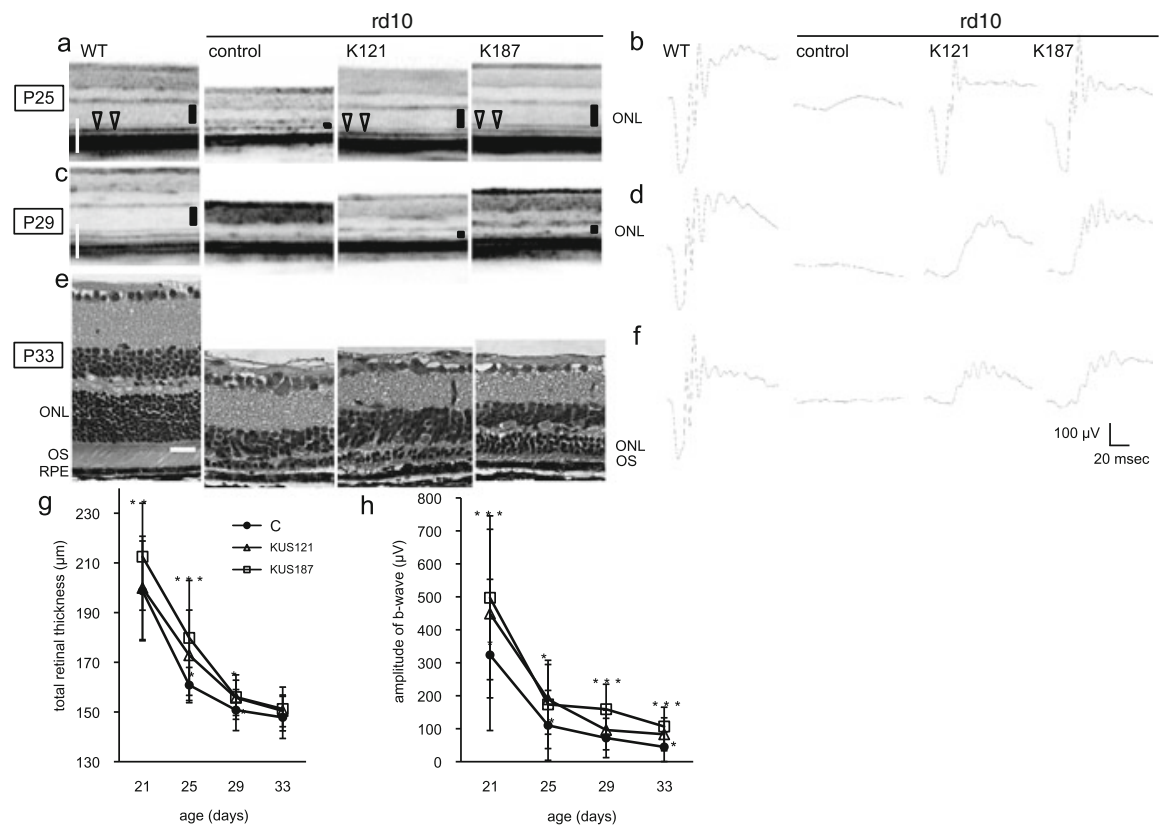

Fig. 4 In vivo efficacies of Kyoto University Substances (KUSs) in the rd10 mouse model of retinitis pigmentosa (Modified from Ikeda et al. [22]). (a, c) Representative live sectional images (vertical sections) by spectral-domain optical coherence tomography (SD-OCT) of retinas in 25-day-old (a) and 29-day-old (c) normal C57BL/6 mice (WT) and rd10 mice, administered KUS $121(n=17)$, KUS187 $(n=21)$, or saline $(n=18)$ as a control. Vertical bars in the images indicate the thickness of the outer nuclear layer (ONL). Note that the ONL was barely detectable in salinetreated control rd10 mice. (b, d, f) Electroretinograms of 25-day-old (b), 29-day-old (d), and 33-day-old (f) normal C57BL/6 mice (WT) and rd10 mice, administered KUSs or saline. (e) HE-stained retinas of 33-day-old normal C57BL/6 mice (WT) and rd10 mice, administered KUSs or saline. Scale bars (shown by white color), $100 \mu \mathrm{m}$ in (a) and (c); $20 \mu \mathrm{m}$ in (e). (g, h) Timedependent changes of total retinal thickness $(\mathbf{g})$ and b-wave amplitude in dark-adapted electroretinograms (h) in rd10 mice administered KUSs or saline (C). $* P<0.05$, $* * P<0.01$, *** $P<0.005$ vs. saline (Dunnett's test). Error bars indicate standard deviations. $O S$ outer segment, RPE retinal pigment epithelium

detected in SD-OCT images, and electroretinogram records were almost flat in control rd10 mice. In the age-matched KUS-treated rd10 mice, the ONL, although thin, and an electroretinogram response were still observed (Fig. 4c, d).

By histological examination, at age 33 days, the ONL in the control rd10 mice consisted of only 1-2 rows of cells, but there remained 5-6 rows of cells in the ONL in the KUS-treated rd10 mice (Fig. 4e). In KUS-treated mice but not in control rd10 mice, the outer segment of the photoreceptors was observed (Fig. 4e). The electroretinogram was non-recordable in the control rd10 mice, but small b-wave and oscillatory potentials were observed in the KUS-treated rd10 mice (Fig. 4f). Timedependent changes in total retinal thickness measured on SD-OCT images (Fig. 4g) and in b-wave amplitudes of dark-adapted electroretinograms (Fig. 4h) showed that 
KUS treatments had the potential to prevent or delay the disease progression. When examined by electron microscopy, the outer segment of the control rd10 mouse retina was mostly disarranged at the age of 21 days, whereas that of the KUS-treated rd10 mouse retina was regularly arranged [22].

\section{Discussion}

We showed that KUSs, new compounds developed as ATPase inhibitors of VCP, have novel functions as "VCP modulators" or "ATP regulators" without apparent inhibition of cellular VCP functions. These new "ATP regulators" have strong neuroprotective effects in vivo on retinal photoreceptor cells. The efficacies were apparently correlated with their abilities to suppress ER stress. From the evidence that KUSs could prevent the decrease in the cellular ATP level in response to several cell death-inducing insults and thereby prevent cell death in pathological conditions, and the evidence that the prevention of early cell death could in turn prevent or delay the deterioration of the affected organs, we posit that a reduction of ATP levels is a common feature in the affected organs of individuals with incurable disorders involving early cell death. Because many proteins require ATP, a reduction of ATP levels would contribute to a functional decline in affected cells or organs in the early stages of the disease. Reducing ATP consumption by way of KUSs and/or enhancing ATP generation by yet-unknown compounds would be a novel strategy to retard these processes and thus to prevent or retard the progression of clinical manifestations [22].

Open Access This chapter is distributed under the terms of the Creative Commons Attribution Noncommercial License, which permits any noncommercial use, distribution, and reproduction in any medium, provided the original author(s) and source are credited.

\section{References}

1. Lin JH, Lavail MM (2010) Misfolded proteins and retinal dystrophies. Adv Exp Med Biol 664:115-121

2. Jiang H, Xiong S, Xia X (2014) Retinitis pigmentosa-associated rhodopsin mutant T17M induces endoplasmic reticulum (ER) stress and sensitizes cells to ER stress-induced cell death. Mol Med Rep 9:1737-1742

3. Higashiyama $\mathrm{H}$ et al (2002) Identification of ter 94 , Drosophila VCP, as a modulator of polyglutamine-induced neurodegeneration. Cell Death Differ 9:264-273

4. Kakizuka A (2008) Roles of VCP in human neurodegenerative disorders. Biochem Soc Trans 36:105-108

5. Watts GD et al (2004) Inclusion body myopathy associated with Paget disease of bone and frontotemporal dementia is caused by mutant valosin-containing protein. Nat Genet 36:377-381

6. Johnson JO et al (2010) Exome sequencing reveals VCP mutations as a cause of familial ALS. Neuron 68:857-864 
7. Manno A, Noguchi M, Fukushi J, Motohashi Y, Kakizuka A (2010) Enhanced ATPase activities as a primary defect of mutant valosin-containing proteins that cause inclusion body myopathy associated with Paget disease of bone and frontotemporal dementia. Genes Cells 15:911-922

8. Stolz A, Hilt W, Buchberger A, Wolf DH (2011) Cdc48: a power machine in protein degradation. Trends Biochem Sci 36:515-523

9. Meyer H, Bug M, Bremer S (2012) Emerging functions of the VCP/p97 AAA-ATPase in the ubiquitin system. Nat Cell Biol 14:117-123

10. Wolf DH, Stolz A (2012) The Cdc48 machine in endoplasmic reticulum associated protein degradation. Biochim Biophys Acta 1823:117-124

11. Hirabayashi $\mathrm{M}$ et al (2001) $\mathrm{VCP} / \mathrm{p} 97$ in abnormal protein aggregates, cytoplasmic vacuoles, and cell death, phenotypes relevant to neurodegeneration. Cell Death Differ 8:977-984

12. Kobayashi T, Tanaka K, Inoue K, Kakizuka A (2002) Functional ATPase activity of p97/valosin-containing protein (VCP) is required for the quality control of endoplasmic reticulum in neuronally differentiated mammalian PC12 cells. J Biol Chem 277:47358-47365

13. Chou TF et al (2011) Reversible inhibitor of $\mathrm{p} 97$, DBeQ, impairs both ubiquitin-dependent and autophagic protein clearance pathways. Proc Natl Acad Sci U S A 108:4834-4839

14. Zinszner $\mathrm{H}$ et al (1998) CHOP is implicated in programmed cell death in response to impaired function of the endoplasmic reticulum. Genes Dev 12:982-995

15. Kim R, Emi M, Tanabe K, Murakami S (2006) Role of the unfolded protein response in cell death. Apoptosis 11:5-13

16. Naidoo N (2009) ER and aging-Protein folding and the ER stress response. Ageing Res Rev 8:150-159

17. Gardner BM, Pincus D, Gotthardt K, Gallagher CM, Walter P (2013) Endoplasmic reticulum stress sensing in the unfolded protein response. Cold Spring Harb Perspect Biol 5:a013169

18. Gorman AM, Healy SJM, Jager R, Samali A (2012) Stress management at the ER: regulators of ER stress-induced apoptosis. Pharmacol Ther 134:306-316

19. Chang B et al (2002) Retinal degeneration mutants in the mouse. Vision Res 42:517-525

20. Ojima Y et al (2010) Restoration of outer segments of foveal photoreceptors after resolution of central serous chorioretinopathy. Jpn J Ophthalmol 54:55-60

21. Oishi A et al (2010) The significance of external limiting membrane status for visual acuity in age-related macular degeneration. Am J Ophthalmol 150:27-32

22. Ikeda $\mathrm{HO}$ et al (2014) Novel VCP modulators mitigate major pathologies of rd10, a mouse model of retinitis pigmentosa. Sci Rep 4:5970 